\title{
Possible changes in hematological parameters of the patients with obstructive sleep apnea
}

\author{
Deniz Doğan ${ }^{1}$, Ramazan Öcal ${ }^{2}$ \\ (1) University of Health Sciences, Gulhane Medical Faculty, Department of Chest Diseases, Ankara, Turkey \\ (2) University of Health Sciences, Gulhane Medical Faculty, Department of Hematology, Ankara, Turkey
}

Date submitted:

Nov 05, 2018

Date accepted:

Feb 06, 2019

Online publication date:

September 15, 2019

\section{Corresponding Author: \\ Ramazan Öcal \\ University of Health Sciences, \\ Gulhane Medical Faculty, \\ Department of Chest Diseases, \\ Ankara, Turkey \\ drocal@gmail.com}

Keywords: obstructive sleep apnea, erythrocytosis, hemoglobin, red blood cell, hematocrit, body mass index.

\begin{abstract}
Aims:Obstructive sleep apnea (OSA) may cause secondary erythrocytosis due to sustained hypoxemia. However, controversial findings have also been reported. The present study investigated the relationship between hematological parameters and OSA severity.

Methods:This retrospective study included individuals who underwent a polysomnography test. Patients were divided into 4 groups based on their apnea-hypopnea index: normal, mild, moderate, and severe OSA. Hemoglobin (HGB), red blood cell (RBC), hematocrit (HCT), red cell distribution width (RDW), mean corpuscular volume (MCV), mean corpuscular hemoglobin $(\mathrm{MCH})$, mean corpuscular hemoglobin concentration (MCHC), white blood cell (WBC), \#neutrophil, \#lymphocyte, erythrocyte sedimentation rate (ESR) and serum lactate dehydrogenase (LDH) levels were evaluated.

Results:The total sample included 400 patients. Mean HGB, RBC, HCT and ESR were significantly higher in severe OSA group ( $p=0.05, p=0.05, p=0.02$ and $p=0.01$, respectively). Mean MCV was significantly lower in subjects with OSA $(p=0.01)$. RDW-CV showed association to the OSA severity $(p=0.01)$, but RDW-SD was similar in OSA groups ( $p=0.07)$. Mean AHI, ESR and LDH were higher in obese patients ( $p=0.001, p=0.01, p=0.005$, respectively). While mean HGB, RBC, HCT values were higher in obese cases $(p=0.05, p=0.05, p=0.01)$, mean MCV was higher in nonobese individuals $(p=0.01)$.

Conclusions:Our results suggest that the severity of OSA can modify HGB and HCT levels in the complete blood count. Whether the magnitude of such a stimulation is high enough to cause secondary polycythemia needs further studies.
\end{abstract}

\section{Introduction}

Secondary polycythemia, an increase in red blood cell mass, is classified as primary and secondary due to circulating erythropoiesis-stimulating factors, usually erythropoietin (Epo). Secondary polycythemia would more accurately be termed secondary erythrocytosis or erythrocythemia, as those terms specifically denote increased red blood cells. While the term polycythemia predominantly defines polycythemia vera, in which there are elevated levels of all three peripheral blood cell lines: red blood cells, white blood cells, and platelets, secondary erythrocytosis is a more suitable term for increase in cells associated with erythrocytes. A well described etiology for secondary erythrocytosis includes sustained hypoxemia caused by a variety of conditions including chronic obstructive pulmonary disease, tobacco use, obstructive sleep apnea, obesity hypoventilation syndrome (1-3).

Obstructive sleep apnea (OSA) is a chronic disease characterized by repetitive collapse of the upper airway during sleep. The episodic hypoxemia and re-oxygenation observed in OSA induces the tonic elevation of sympathetic nervous system, ox- idative stress, hypercoagulability, endothelial dysfunction and systemic inflammation. It is believed that co-morbidities observed in OSA based on these local and systemic inflammations and recurrent hypoxemia $(4,5)$.

Due to the fact that OSA is a common condition associated with a decrease in oxyhemoglobin saturation during sleep, various hematological guidelines suggest an assessment for OSA in the evaluation of secondary erythrocytosis etiology. However, whether untreated OSA leads to clinically significant erythrocytosis remains controversial $(6,7)$.

In this study, we aimed to identify the relationship between hematological parameters and OSA severity, and also the possible pro-inflammatory effects of OSA severity and obesity. Furthermore, we also examined whether OSA is associated with erythrocytosis.

\section{Methods}

This retrospective study was approved by the local ethics committee. Using the medical records, patients who underwent all night polysomnography (PSG) test in our clinic between 
June 2013 and June 2016 due to sleep apnea and/or sleep disorder complaints were identified. The exclusion criteria were insufficient data of demographics and comorbidities, presence of chronic diseases (cardiovascular diseases, hematological diseases, oncological diseases, kidney diseases, diabetes mellitus, thyroid pathologies, significant allergic reactions), drug use, infections, and lack of adequate sleep efficiency. Age, gender, body-mass index (BMI), apnea-hypopnea index $(\mathrm{AHI})$, complete blood count values which were performed 1-3 days before PSG test including hemoglobin (HGB), red blood cell (RBC), hematocrit (HCT), red cell distribution width (RDW), RDW-coefficient of variation (RDW-CV), RDW-standard deviation (RDW-SD), mean corpuscular volume (MCV), mean corpuscular hemoglobin $(\mathrm{MCH})$, mean corpuscular hemoglobin concentration ( $\mathrm{MCHC}$ ), white boold cell (WBC), absolute neutrophil count (\#neutrophil), absolute lymphocyte count (\#lymphocyte), erythrocyte sedimentation rate (ESR), and serum lactate dehydrogenase (LDH) levels were recorded.

Patients were divided into 4 groups according to their $\mathrm{AHI}$

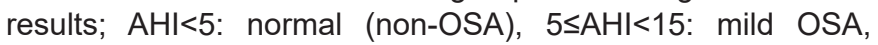
$15 \leq \mathrm{AHI}<30$ : moderate OSA, $\mathrm{AHI} \geq 30$ : severe OSA (8). According to this classification two main groups were formed as nonOSA (normal) and OSA. Patients were also grouped according to $\mathrm{BMI}$; obese and non-obese. The grouping was based on BMI classification of World Health Organization; BMl<30: nonobese, $\mathrm{BMI} \geq 30$ : obese (9).

\section{Statistical analyses}

The data were analyzed by SPSS for Mac 20.0 package program (SPSS Inc, Chicago, IL, USA). Frequency and percentage for discrete data, mean \pm SD for continuous variables were used in descriptive statistics. The normality of the continuous variables was analyzed with the Kolmogorov-Smirnov test.
Mean values with parametric distribution between groups were analyzed by Student's T test and ANOVA. Mean values with non-parametric distributions were compared by Mann-Whitney $\mathrm{U}$ and Kruskal-Wallis $\mathrm{H}$ tests. Correlations were calculated using the Pearson test and Spearman test where appropriate. $\mathrm{P}$-value less than 0.05 was considered as statistically significant.

\section{Results}

The sample size included 400 individuals. 308 (87\%) cases were diagnosed with OSA due to $\mathrm{AHI}$ and 92 were non-OSA (normal). 366 (91.5\%) patients were male and 34 (8.5\%) were female. Number of cases (n), mean values of age, BMI, ESR, LDH, HGB, HCT, RDW-CV, RDV-SD, MCV, MCH, MCHC values of each $\mathrm{AHI}$ group are shown in Table 1. Polycythemia was observed in 16 OSA cases (5.2\%). The differences between groups were significant for mean HGB, RBC, HCT, RDW-CV, MCV and ESR $(p=0.05, p=0.05, p=0.02, p=0.01, p=0.01$, and $p=0.01$, respectively) (Table 1 ). Parameters related to erythrocyte serial (HGB, RBC, HCT) were significantly higher in severe OSA group $(p=0.05, p=0.05$, and $p=0.02)$. MCV was significantly lower in OSA cases, especially in severe OSA group $(p=0.01)$. While RDW-CV showed a relationship with OSA severity, RDW-SD did not show any significance difference between OSA groups ( $p=0.01$ and $p=0.07$ respectively). WBC, \#neutrophil and \#lymphocyte counts did not show any significance difference between groups (Table 1).

According to BMI, 120 cases (30\%) were obese and 240 $(70 \%)$ were non-obese. The mean age was $42.57 \pm 12.73$ (23$70)$ in obese group and $36.29 \pm 12: 45$ (16-78) in non-obese group. The mean AHI values were $30.86( \pm 24.17)$ and 20.86 $( \pm 21.78)$ in these two groups, respectively. The mean age and $\mathrm{AHI}$ values were significantly higher in the obese group

Table 1: Mean values ( \pm standard deviation) of data due to OSA severity groups.

\begin{tabular}{|c|c|c|c|c|c|}
\hline & $\begin{array}{c}\text { Normal } \\
\text { (non-OSA) } \\
(\mathrm{AHI}<5)\end{array}$ & $\begin{array}{c}\text { Mild OSA } \\
(5 \leq \mathrm{AHI}<15)\end{array}$ & $\begin{array}{l}\text { Moderate OSA } \\
(15 \leq \mathrm{AHI}<30)\end{array}$ & $\begin{array}{l}\text { Severe OSA } \\
(\mathrm{AHI} \geq 30)\end{array}$ & $\mathbf{p}$ \\
\hline n (\%) & $92(23 \%)$ & $92(23 \%)$ & $92(23 \%)$ & $124(31 \%)$ & - \\
\hline Age (year) & $34.8 \pm 12.5$ & $39.0 \pm 11.0$ & $39.7 \pm 14.1$ & $38.8 \pm 13.1$ & NS \\
\hline $\operatorname{BMI}\left(\mathrm{kg} / \mathrm{m}^{2}\right)$ & $26.2 \pm 3.0$ & $27.9 \pm 3.6$ & $28.2 \pm 3.6$ & $29.6 \pm 3.9$ & NS \\
\hline HGB (g/L) & $13.8 \pm 2.5$ & $14.2 \pm 2.3$ & $14.7 \pm 1.9$ & $16.1 \pm 2.0$ & 0.05 \\
\hline RBC ( $x 103$ cell $/ \mu L)$ & $4.8 \pm 1.2$ & $4.9 \pm 1.1$ & $5.3 \pm 1.6$ & $5.9 \pm 1.4$ & 0.05 \\
\hline HCT (\%) & $43.3 \pm 5.3$ & $44.5 \pm 4.2$ & $45.7 \pm 5.4$ & $50.1 \pm 3.7$ & 0.02 \\
\hline RDW-CV (\%) & $12.5 \pm 1.5$ & $13.2 \pm 2.3$ & $14.7 \pm 1.2$ & $15.3 \pm 1.9$ & 0.01 \\
\hline RDW-SD (fL) & $40.5 \pm 3.5$ & $41.3 \pm 4.1$ & $42.3 \pm 3.7$ & $42.9 \pm 4.2$ & NS \\
\hline MCV(fL) & $89.9 \pm 4.2$ & $86.3 \pm 4.5$ & $85.4 \pm 3.4$ & $81.8 \pm 3.1$ & 0.01 \\
\hline $\mathrm{MCH}(\mathrm{pg})$ & $29.2 \pm 2.5$ & $29.7 \pm 2.7$ & $30.2 \pm 3.8$ & $31.1 \pm 2.9$ & NS \\
\hline $\operatorname{MCHC}(g / L)$ & $33.3 \pm 3.1$ & $33.8 \pm 2.8$ & $34.3 \pm 2.3$ & $34.6 \pm 2.1$ & NS \\
\hline WBC(x103 cell/ $/ \mu L)$ & $6635 \pm 1457$ & $6918 \pm 1636$ & $6695 \pm 1227$ & $7037 \pm 1634$ & NS \\
\hline \#neut. ( $\times 103$ cell $/ \mu L)$ & $3677 \pm 1194$ & $3850 \pm 1325$ & $3608 \pm 931$ & $3915 \pm 1146$ & NS \\
\hline 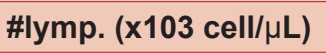 & $2234 \pm 514$ & $2307 \pm 618$ & $2271 \pm 528$ & $2408 \pm 587$ & NS \\
\hline $\operatorname{ESR}(\mathrm{mm} / \mathrm{h})$ & $5.4 \pm 3.92$ & $7.7 \pm 3.83$ & $7.2 \pm 4.43$ & $8.11 \pm 4.24$ & 0.01 \\
\hline LDH(U/L) & $266.0 \pm 100.7$ & $274.7 \pm 78.2$ & $236.0 \pm 77.6$ & $254.1 \pm 75.2$ & NS \\
\hline \multicolumn{6}{|c|}{$\begin{array}{l}\text { OSA: obstructive sleep apnea, AHI: apnea-hypopnea index, BMI: body mass index, HGB: hemoglobin, RBC: red blood cell, HCT: hematocrit, RDW: red cell distribution width, RDW-CV: red } \\
\text { cell distribution width-coefficient of variation, RDW-SD: red cell distribution width-standard deviation, MCV: mean corpuscular volume, MCH: mean corpuscular hemoglobin, MCHC: mean } \\
\text { corpuscular hemoglobin concentration, WBC: white blood cell, \#neutrophil: absolute neutrophil count, \#lymphocyte: absolute lymphocyte count, ESR: erythrocyte sedimentation rate, LDH: } \\
\text { serum lactate dehydrogenase. }\end{array}$} \\
\hline
\end{tabular}


( $p=0.001, p=0.004$, respectively)

After the excluding the non-OSA (normal) cases, data of 308 patients diagnosed with OSA were analyzed separately. When the results of obese and non-obese OSA patients were compared, the mean $\mathrm{AHI}$ values were significantly higher in obese patients $(p=0.001)$. The mean AHI values were $34.6( \pm 24.17)$ and $28.1( \pm 21.6)$ in these two groups, respectively. Hematological parameters also showed some differences across different BMI groups in the OSA group. While mean HGB, RBC, HCT values were significantly higher in the obese OSA cases, mean MCV was significantly higher in non-obese OSA cases $(p=0.05$, $p=0.05, p=0.01$ and $p=0.01$, respectively). ESR and LDH levels were also higher in obese OSA group $(p=0.01, p=0.005$, respectively). The mean values of OSA patients and its subgroups according to BMI are summarized in Table 2 (Table 2).

When the data of 53 obese+OSA patients examined in detail, we determined that $13(24.5 \%)$ patients had mild OSA, 14 $(26.5 \%)$ had moderate, $26(49 \%)$ had severe OSA. The numbers for 101 non-obese+OSA patients were 33 (32.7\%), 32 $(31.7 \%)$ and $36(35.6 \%)$, respectively. The frequency of severe OSA was higher in obese patients than non-obese patients.

There were positive correlations between $\mathrm{AHI}$ with ESR, BMI, HGB, RBC, HCT and RDW-CV. However, MCV was negatively correlated with AHI, BMI, HGB, RBC, HCT and RDW-CV (Table 3).

\section{Discussion}

OSA, is a chronic pathology with an increasing awareness in the population especially by means of widespread sleep tests. Although OSA may be observed in both genders and in a wide range of ages, demographic data indicates that frequency and severity of OSA increase in middle-aged and older cases (4, 5, 10). According to national health and nutrition survey, a comprehensive study which was performed in United States (US) between the years $2005-2008$ on 10.352 subjects, the mean age of the cases who were evaluated to have high risk for OSA was $55.2 \pm 0.54$ years (11). The mean age of OSA cases in our study was younger, but we think that this situation resulted from the exclusion of the patients with co-morbidities such as cardiovascular diseases which are observed more frequently in older age.

There are many studies about the relationship of OSA and obesity $(12,13)$. Salerno et al. stated that mean BMI $(37 \pm 9)$ was significantly higher in OSA cases compared to the healthy controls (14). Our results about the relationship of BMI and OSA are also consistent with the literature. Our most powerful evidences in this regard were higher mean BMI in OSA patients and the significant correlation between $\mathrm{BMI}$ and $\mathrm{AHI}$ in OSA cases.

Another important issue about the role of obesity in OSA is that obesity is the main origin of inflammation in these patients. It is known that obesity itself is also directly stimulates inflammation. In addition to leptin, a pro-fibrogenic adipokine released from the adipose tissue, many pro-inflammatory cytokines such as TNF- $\alpha$, IL-6, IL-8 have been associated with OSA severity (15). Significant decreases were observed after weight loss in these markers and also in $\mathrm{AHI}(16,17)$. Yardim-Akaydin et al (18) demonstrated CRP, fibrinogen and ESR at higher levels in OSA cases with obesity and/or metabolic syndrome. In another study, ESR was not higher in general population of OSA patients and was significantly higher in only obese OSA cases (19). Considering that significant positive correlation observed between BMI and ESR in OSA cases and the significant elevation of ESR and LDH in obese cases compared to nonobese cases, it can be said that our results are consistent with literature.

There are many previous studies about the association of ESR and OSA. In the study by Peled et al (20), ESR, CRP and

\begin{tabular}{|c|c|c|c|c|}
\hline & OSA & Obese+OSA & Non-obese+OSA & $\mathbf{p}$ \\
\hline n (\%) & $308(100 \%)$ & $106(34.4 \%)$ & $202(65.6 \%)$ & - \\
\hline Age (year) & $39.2 \pm 12.7$ & $42.9 \pm 13.2$ & $37.2 \pm 12.1$ & NS \\
\hline $\mathrm{AHI}$ & $30.3 \pm 22.3$ & $34.6 \pm 23.1$ & $28.1 \pm 21.6$ & 0.001 \\
\hline HGB (g/L) & $15.1 \pm 2.4$ & $15.5 \pm 2.4$ & $14.9 \pm 2.4$ & 0.05 \\
\hline RBC ( $\times 103$ cell/ $/ \mu L)$ & $5.4 \pm 1.6$ & $5.9 \pm 1.4$ & $5.1 \pm 1.8$ & 0.05 \\
\hline HCT (\%) & $47.1 \pm 4.5$ & $49.3 \pm 4.2$ & $45.9 \pm 4.6$ & 0.01 \\
\hline RDW-CV (\%) & $14.4 \pm 1.5$ & $15.1 \pm 1.6$ & $14.1 \pm 1.7$ & 0.02 \\
\hline RDW-SD (fL) & $42.2 \pm 4.1$ & $42.4 \pm 4.1$ & $42.1 \pm 4.1$ & NS \\
\hline MCV (fL) & $84.2 \pm 3.8$ & $82.2 \pm 3.7$ & $85.2 \pm 3.6$ & 0.01 \\
\hline $\mathrm{MCH}(\mathrm{pg})$ & $30.4 \pm 3.2$ & $31.5 \pm 3.3$ & $29.8 \pm 3.4$ & NS \\
\hline MCHC (g/L) & $34.3 \pm 3.2$ & $35.3 \pm 3.6$ & $33.8 \pm 3.1$ & NS \\
\hline WBC (x103 cell/ $/ \mu \mathrm{L})$ & $6900 \pm 1527$ & $7042 \pm 1608$ & $6225 \pm 1485$ & NS \\
\hline \#neut. ( $x 103$ cell/ $\mu L)$ & $3810 \pm 1152$ & $3834 \pm 1124$ & $3797 \pm 1172$ & NS \\
\hline \#lymp. ((x103 cell/ $/ \mathrm{L})$ & $2326 \pm 570$ & $2343 \pm 664$ & $2318 \pm 517$ & NS \\
\hline ESR (mm/h) & $7.7 \pm 4.2$ & $9.1 \pm 4.08$ & $6.9 \pm 4.3$ & 0.01 \\
\hline LDH (U/L) & $254.6 \pm 76.9$ & $265.1 \pm 67.3$ & $249.5 \pm 82.5$ & 0.005 \\
\hline
\end{tabular}


Table 3: Significant correlations between parameters in OSA cases.

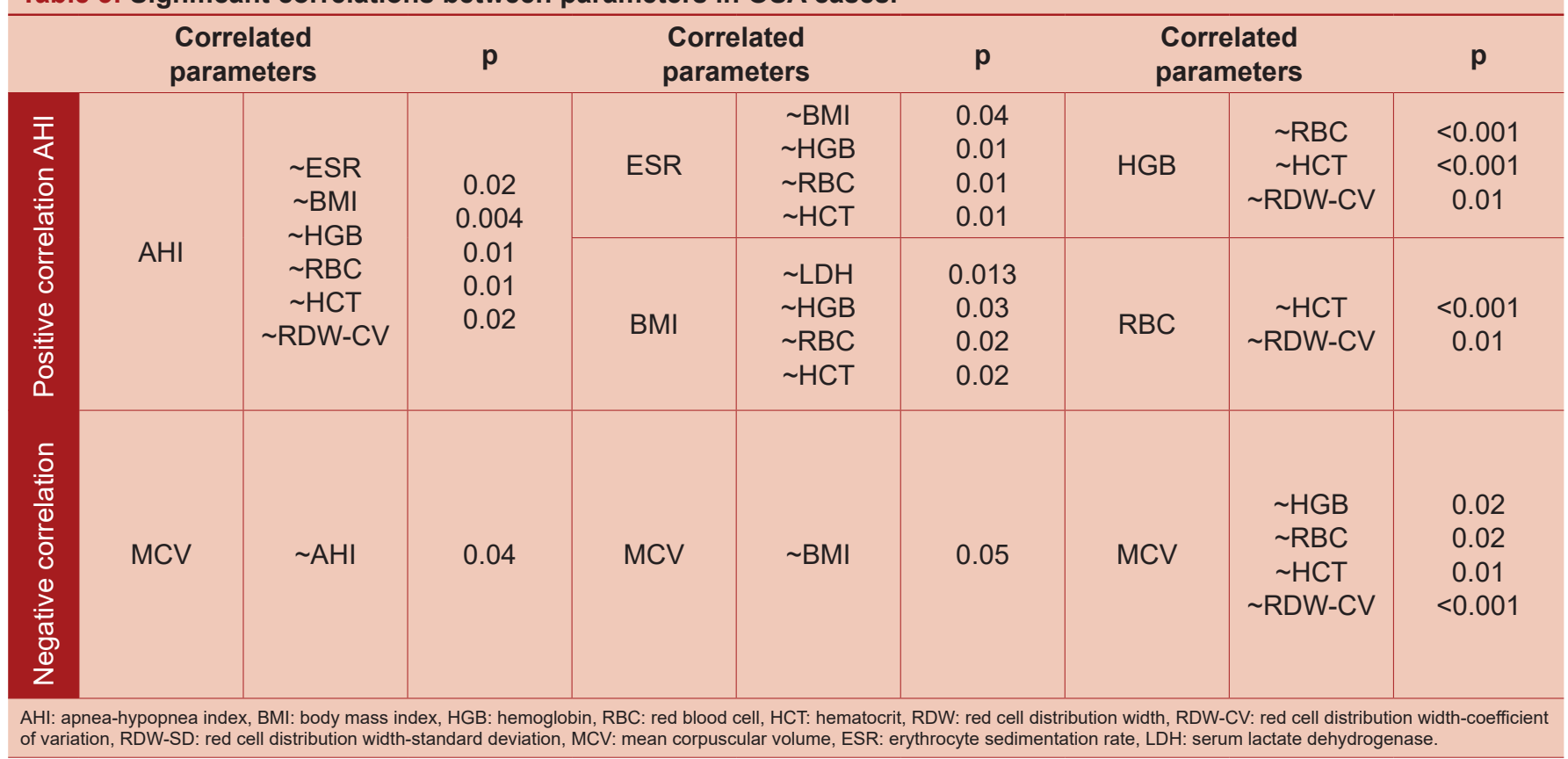

fibrinogen were found to be higher in OSA cases and a positive correlation was found between $\mathrm{AHI}$ and CRP. Dikmenogluet al (21) also demonstrated that ESR, CRP and fibrinogen levels were significantly higher in OSA cases. Our results are also consistent with the previous literature. We observed that mean ESR level was significantly higher in the OSA group than non-OSA group. In addition, the significant positive correlations between ESR with $\mathrm{AHI}$ and BMI in OSA cases also strengthened our findings. In the light of all these findings, it can be suggested that increased ESR is a useful predictor of potential co-morbidities that may accompany OSA.

$\mathrm{LDH}$ is an intracellular enzyme which presents in every cell in the body. LDH levels generally increase in plasma due to cell damage and cell turnover. This increase carries prognostic value in situations such as hemolytic anemia, myocardial infarction, hepatocellular injury, solid tumors and hematologic malignancies like lymphoma (22). There is limited number of studies about the relationship between LDH and OSA in the literature. In a previous study, investigating the effects of OSA on glucose regulation and liver damage in non-diabetic cases, positive correlations were found between serum LDH with CRP, insulin levels and insulin resistance (23). On the other hand, according to the US national screening results there was no significant differences in mean LDH levels between high and Iow OSA risk groups (11). In our study, we investigated the possible relationship of LDH both with OSA severity and the levels of other blood cells; and demonstrated that mean LDH value was significantly higher in obese OSA patients and a significant positive correlation was present between LDH and BMI in OSA cases. Thus, we suggest that tissue damage and inflammation are discernable in OSA and the role of LDH in OSA is related to obesity particularly.

In terms of the role of blood cells in OSA, different statements are available. There are some studies in the literature on the relationship between OSA and erythrocytosis. It is generally accepted that OSA may cause secondary polycythemia by inducing erythrocytosis. In Hoffstein et al.'s (24) study, one of the oldest studies on this subject, it was stated that intermittent nocturnal hypoxemia during apnea episodes does not lead to polycythemia, but is associated with elevations in hematocrit value. In later studies with larger number of cases, this issue has been discussed but contradictory results have also been found. In a study of 1604 cases, the authors stated that erythrocytosis is not associated with OSA severity, but rather with hypoxemia. It was also suggested that nocturnal oximetry and polysomongraphy may be used as diagnostic tools in this regards (25). On the other hand, Gangaraju et al. (26) reported that polycythemia was less common than predicted in OSA, and no correlation was found between OSA severity and hemoglobin levels. In our study, polycythemia was not frequent (5.2\%), partially consistent with Gangaraju's study. However, in our results, a positive correlation was present between OSA severity assessed by $\mathrm{AHI}$ and HGB, HCT, RBC values; and also a significant difference was observed between OSA severity groups. According to our results it can be stated that the increase in apnea periods, albeit rarely, leads to polycythemia stimulating erythrocytosis.

Some data are available in the literature about the relationship of RDW values and OSA. In their retrospective study, Yousef and Alkhiary (27) reported that higher RDW, MPV, PDW and platelet counts were related to OSA; RDW was positively correlated with $\mathrm{AHI}$, oxygen desaturation index, Epworth sleepiness scale, and negatively correlated with minimum oxygen saturation and rapid eye movement sleep. In the study of Sunnetcioglu et al. (28), RDW was found to be significantly higher in severe OSA with cardiovascular disorders. In our study, RDW-CV and RDW-SD were examined separately and there was no significant difference between groups in terms of RDW$\mathrm{SD}$. On the other hand, RDW-CV was evaluated as an associated marker of both OSA severity and obesity. In this respect, we think that our study contributes to the current literature as there are reports about the relationship of OSA severity and MCV, a component of the RDW calculation $(29,30)$. Likewise, we observed negative correlations between MCV and HGB, $\mathrm{HCT}, \mathrm{RBC}$; and we suggest this may be related to increased consumption of iron due to erythrocytosis. 
RDW is measured by two methods which are termed as "Red cell distribution width - coefficient of variation (RDW-CV)" and the "Red cell distribution width - standard deviation (RDW-SD)". The RDW-CV is a calculation based on both the mean cell size of RBC and the width of the distribution curve. It is calculated by dividing the standard deviation by the MCV and multiplying by 100 to convert to a percentage. While a normal range for the RDW-CV is approximately $11.0-15.0 \%$, RDW-SD ranges between $40.0-55.0 \mathrm{fL}$. The most notable feature of RDW-CV is that it reflects the variability of not only the width of the distribution curve but also the MCV of the red cell population (31-33). The negative correlation of MCV with RDW-CV and the fact that RDW-SD does not show a significant relationship with OSA weight or MCV in our cases, can be explained by this situation. In other words, the erythrocytosis in OSA may reduce the MCV due to consumption and affects the width of the distribution curve relatively, but not absolutely.

Being a single-center retrospective data review is the most important limitation of our study. The fact that we could not measure Epo due to retrospective design prevents us from expressing the etiopathogenesis of polycythemia as primary or secondary in our cases.

In conclusion, according to our study, OSA severity may affect hemoglobin, hematocrit levels in the complete blood count by stimulating erythocytosis in the bone marrow, but this situation may rarely result in secondary polycythemia. However, in the etiology of secondary polycythemia, OSA should be kept in mind as an underlying disease.

\section{Acknowledgments}

Authorship contributions: Concept: DD, RO; Design: DD, RO; Supervision: DD, RO; Resources: DD; Materials: DD; Data collection and/or processing: DD; Analysis and/or interpretation: DD, RO; Writing: DD, RO; Critical review: DD, RO.

\section{Conflict of Interest}

The authors declare that they do not have anything to disclose regarding conflict of interest with respect to this manuscript.

\section{References}

1. Galeas JN, Yu Y, Polineni R, et al. Polycythemia Vera vs. Secondary Erythrocytosis Mortality Outcome in a Multiracial Inner City Cohort. Blood. 2015;126(23):5183.

2. Narváez PA, Mohrenberger CJ, Baena EM, et al. Erythrocytosis in patients with obstructive sleep apnea. European Respiratory Journal. 2014;44 (Suppl 58): P2210.

3. Keohane,C, McMullin MF. The diagnosis and management of erythrocytosis. BMJ. 2013(Nov 18);347:f6667.

4. Grote L, Hedner J, Peter JH. Sleep-related breathing disorder is an independent risk factor for uncontrolled hypertension. J Hypertens. 2000;18(6):679-685.

5. Kokturk O, Ciftci TU, Mollarecep E, Ciftci B. Elevated C-reactive protein levels and increased cardiovascular risk in patients with obstructive sleep apnea syndrome. Int Heart J. 2005;46(5):801-809.

6. Nistico A, lliescu EA, Fitzpatrick M, White CA. Polycythemia due to obstructive sleep apnea in a patient on hemodialysis. Hemodial Int. 2010;14(3):333-336.
7. Kurt OK, and Yildiz N. The importance of laboratory parameters in patients with obstructive sleep apnea syndrome. Blood Coagul. Fibrinolysis Int. J. Haemostasis Thromb. 2013;24(4):371-374.

8. Berry RB, Budhiraja R, Gottlieb DJ, et al. Rules for scoring respiratory events in sleep: update of the 2007 AASM Manual for the Scoring of Sleep and Associated Events. Deliberations of the Sleep Apnea Definitions Task Force of the American Academy of Sleep Medicine. J Clin Sleep Med. 2012;8(5):597-619.

9. WHO 2000 p. 6 Gray DS, Fujioka K. Use of relative weight and Body Mass Index for the determination of adiposity. J Clin Epidemiol. 1991;44(6):545-550.

10. Young T, Palta M, Dempsey J, Skatrud J, Weber S, Badr $\mathrm{S}$. The occurrence of sleep-disordered breathing among middle-aged adults. N Engl J Med. 1993;328(17):12301235.

11. Leyva FJ, Lankapalli RH. Association Among Obstructive Sleep Apnea Risk and Blood Chemistry Changes in the U.S. Population: National Health and Nutrition Examination Survey 2005-2008. The Internet Journal of Epidemiology. 2013; Volume 11, Number 2.

12. Mendes FA, Marone SA, Duarte BB, Arenas AC. Patients with Snoring and Obstructive Sleep Apnea in a University Hospital. Int Arch Otorhinolaryngol. 2014;18(2):142145.

13. Alam I, Lewis K, Stephens JW, Baxter JN. Obesity, metabolic syndrome and sleep. apnoea: All pro-infl ammatory states. Obesity Rev. 2007;8(2):119-127.

14. Salerno FG, Carpagnano E, Guido P, et al. Airway inflammation in patients affected by obstructive sleep apnea syndrome. Respir Med. 2004;98(1):25-28.

15. Dogan D, Ocal N, Aydogan M, et al. Assessment of the role of serum ischemia-modified albumin in obstructive sleep apnea in comparison with interleukin-6.Postgraduate Medicine. 2016;128(6):603-608.

16. Barceló A, Barbé F, Llompart E, et al. Effects of obesity on $\mathrm{C}$-reactive protein level and metabolic disturbances in male patients with obstructive sleep apnea. Am J Med. 2004;117(2):118-121.

17. Barceló A, Barbé F, Llompart E, et al. Neuropeptide $Y$ and leptin in patients with obstructive sleep apnea syndrome: Role of obesity. Am J Respir Crit Care Med. 2005;171(2):183-187.

18. Yardim-Akaydin S, Caliskan-Can E, Firat H, Ardic S, Simsek B. Influence of gender on C-reactive protein, fibrinogen, and erythrocyte sedimentation rate in obstructive sleep apnea. Antiinflamm Antiallergy Agents Med Chem. 2014;13(1):56-63.

19. Min JY, Jang JY, Kim HY, et al. Relationship between the Obstructive Sleep Apnea Syndrome and the Erythrocyte Sedimentation Rate. Clin Exp Otorhinolaryngol. 2009;2(3):126-130.

20. Peled N, Kassirer M, Kramer MR, et al. Increased erythrocyte adhesiveness and aggregation in obstructive sleep apnea syndrome. Thromb Res. 2008;121(5):631636. 
21. Dikmenoğlu N, Ciftçi B, Ileri E, et al. Erythrocyte deformability, plasma viscosity and oxidative status in patients with severe obstructive sleep apnea syndrome. Sleep Med. 2006;7(3):255-261.

22. Granchi C, Capecchi A, Del Frate G, et al. Development and Validation of a Docking-Based Virtual Screening Platform for the Identification of New Lactate Dehydrogenase Inhibitors. Molecules. 2015;20(5):8772-8790.

23. Calvin AD, Albuquerque FN, Lopez-Jimenez F, Somers VK. Obstructive sleep apnea, inflammation, and the metabolic syndrome. Metab Syndr Relat Disord 2009;7(4):271-278.

24. Hoffstein V, Herridge M, Mateika S, Redline S, Strohl KP. Hematocrit levels in sleep apnea. Chest. 1994;106(3):787-791.

25. Nguyen CD, Holty JC. Does untreated obstructive sleep apnea cause secondary erythrocytosis? Respir Med. 2017;130(sep):27-34.

26. Gangaraju R, Sundar KM, Song J, Prchal JT. Polycythemia Is Rarely Caused By Obstructive Sleep Apnea. Blood. 2016;128(22):2444.

27. Yousef A, Alkhiary W. The severity of obstructive sleep apnea syndrome is related to red cell distribution width and hematocrit values. J Sleep Disord Ther. 2015;4(2):192.

28. Sunnetcioglu A, Gunbatar H, Yıldız H. Red cell distribution width and uric acid in patients with obstructive sleep apnea. Clin Respir J. 2018;12(3):1046-1052.

29. Fan Z, Lu X, Long H, Li T, Zhang Y. The association of hemocyte profile and obstructive sleep apnea. J Clin Lab Anal. 2018;23(Sep):e22680 [Epub ahead of print].

30. Uğurlu E, Can İ, Ertürk MS, Akbudak IH, Dursunoğlu N, Özkurt S. Relationship between Hematological Examination, Glucose, HbA1c Level, and Disease Stages in Patients with Obstructive Sleep Apnea Syndrome. Eurasian J Pulmonol. 2018;20(1):33-36.

31. Evans TC, Jehle D. The red blood cell distribution width. J Emerg Med. 1991;9(Suppl 1):71-74.

32. Sökücü SN, Karasulu L, Dalar L, Seyhan EC, Altın S. Can red blood cell distribution width predict severity of obstructive sleep apnea syndrome? J Clin Sleep Med. 2012;15;8(5):521-525.

33. Caporal FA, Comar SR. Evaluation of RDW-CV, RDW$\mathrm{SD}$, and MATH-1SD for the detection of erythrocyte anisocytosis observed by optical microscopy. J Bras Patol Med Lab. 2013;49(5):324-331. 